\title{
As mudanças geradas pela Base Nacional Comum Curricular na abordagem do pensamento algébrico nos anos iniciais do Ensino Fundamental
}

\section{DÉBORA CRISTINA BORBA PEREIRA FAVERO ${ }^{1}$}

ANA LÚCIA MANRIQUE ${ }^{2}$

\begin{abstract}
Resumo
Este artigo trata de uma pesquisa bibliográfica que tem por objetivo analisar dissertações $e$ teses que investigaram o pensamento algébrico nos anos iniciais do Ensino Fundamental. $O$ referencial teórico salienta um modelo dominante de ensino da Álgebra reduzido a uma Aritmética generalizada no Ensino Fundamental, que será bruscamente algebrizado no Ensino Médio. E na atual proposta da BNCC temos a algebrização do currículo desde os anos iniciais. Foram adotadas algumas palavras chave relacionadas ao pensamento algébrico para o levantamento dos trabalhos no banco de teses e dissertações da Coordenação de Aperfeiçoamento de Pessoal do Nível Superior (Capes). Em relação ao tema pensamento algébrico nos anos iniciais do Ensino Fundamental, foram encontrados onze trabalhos sobre sequências didáticas ou a produção escrita dos alunos e dois trabalhos sobre o professor dos anos iniciais. E podemos dizer também que nenhum dos estudos está relacionado à análise de materiais didáticos e existe, nesse levantamento, apenas um trabalho envolvendo a BNCC.
\end{abstract}

Palavras-chave: Pensamento algébrico; Anos Iniciais; BNCC.

\begin{abstract}
This article refers to a bibliographic research that aims to analyze dissertations and thesis that investigated algebraic reasoning in the early years of Elementary School. The theoretical framework highlights a dominant model of teaching Algebra reduced to a generalized Arithmetic in Elementary School, which will be sharply algebraized in High School. And the current proposal of the Base Nacional Comum Curricular (BNCC) (National Common Curricular Base) present an algebraized curriculum since the early years. Some keywords related to algebraic reasoning were adopted to survey the work in the thesis and dissertation database of the Coordenação de Aperfeiçoamento de Pessoal do Nivel Superior (Capes) (Coordination for the Improvement of Higher Education Personnel). Regarding the theme algebraic reasoning in the early years of Elementary School, eleven works were found on didactic sequences or the student's' written production and two works on the teacher of the early years. And we can also say that none of the studies is related to the analysis of teaching materials and there is, in this survey, only one work involving BNCC.
\end{abstract}

Keywords: Algebraic reasoning; Elementary Education; BNCC.

\footnotetext{
1 Pontifícia Universidade Católica de São Paulo. PEPG em Educação Matemática - e-mail: dp.borba@gmail.com.

2 Pontifícia Universidade Católica de São Paulo. PEPG em Educação Matemática - e-mail: analuciamanrique@gmail.com.
} 


\section{Introdução}

Em dezembro de 2017 foi homologada a Base Nacional Comum Curricular (BNCC) referente à Educação Infantil e ao Ensino Fundamental. O documento deverá orientar as propostas pedagógicas e os currículos da Educação Básica, das escolas privadas e públicas de todo o Brasil (BRASIL, 2018). Com isso surge a necessidade de toda uma readequação para seguir a nova legislação vigente, incluindo os materiais didáticos.

O novo documento traz uma divisão da Matemática em cinco unidades temáticas desde os anos iniciais: Números; Álgebra; Geometria; Grandezas e medidas; e Probabilidade e estatística (BRASIL, 2017). O documento que, até então, orientava os currículos eram os Parâmetros Curriculares Nacionais (PCN), que dividiam a Matemática dos anos iniciais em quatro eixos: Números e operações; Espaço e forma; Grandezas e medidas e Tratamento da informação (BRASIL, 1997) (FIGURA 1). A Álgebra, portanto, é instituída com a BNCC como uma unidade da Matemática a ser ensinada desde os anos iniciais.

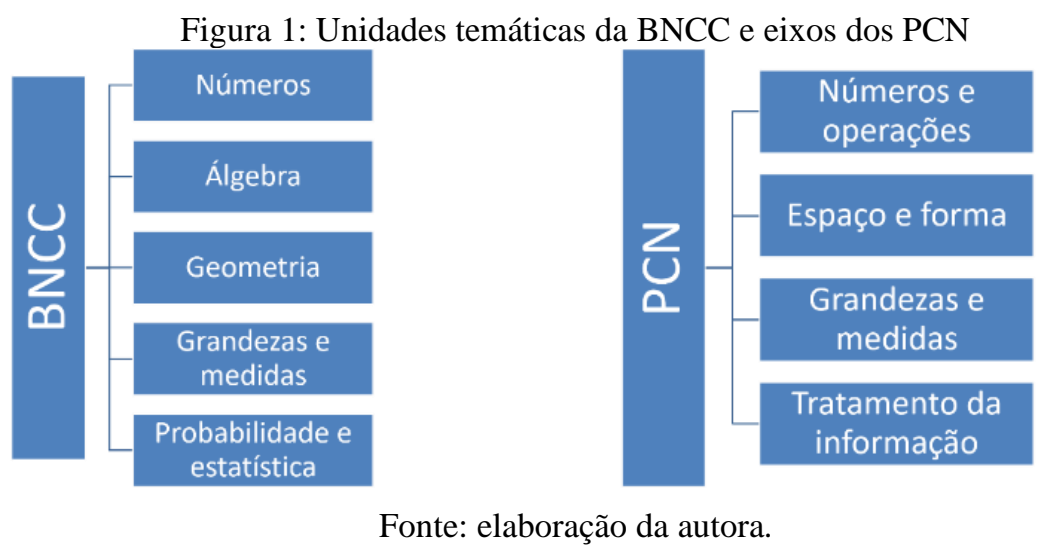

Este artigo apresenta resultados de uma pesquisa bibliográfica que evidencia estudos que abordam o pensamento algébrico nos anos iniciais.

\section{Referencial teórico}

Para tratar de aspectos históricos nos baseamos em Katz (1995) e para os aspectos da Álgebra no currículo nos baseamos nas pesquisas de Chevallard (1984, 1989 e 1990) e alguns de seus seguidores (GASCÓN, 1999; RUIZ, BOSCH, GASCÓN, 2007, 2010, 2015).

Segundo Katz (1995), a obra do islâmico Muhammad ibn Musa al-Khwarizmi datada por volta de 825 é considerada a primeira relacionada a Álgebra. Sua legitimidade é 
concedida por ser a primeira obra a justificar as soluções. A justificativa é feita por meio da Geometria. No entanto, outras obras como o Papyro de Rhind (1650 a. C.), as tábuas de argila babilônicas (1700 a. C.) e o livro chinês Jiuzhang suanshu (200 a.C.) já se ocupavam da tarefa de resolver o que chamamos hoje de equações. Katz (1995) destaca algumas características a respeito da Álgebra presentes em todos esses documentos: a Álgebra sempre foi tratada por meio de resolução de problemas; esses problemas, apesar de concretos, não eram da "vida real"; ela era abordada por meio de ideias de outros campos da Matemática, principalmente da Geometria; e era reservada a uma parte nobre da sociedade.

A evolução histórica da Álgebra pode ser brevemente divida em três etapas: a primeira concebe a Álgebra para resolver equações, principalmente para a solução de problemas aritméticos; a segunda corresponde a estudos de tipos gerais de equações, incluindo o cálculo numérico e constituindo-se como uma área da Matemática; e a terceira é o momento da Álgebra Moderna em que a ferramenta algébrica penetra todos os campos da Matemática, encontra outros campos de aplicação e nos influencia até hoje. Desta forma, é muito difícil encontrar atividades matemáticas que não utilizem o simbolismo algébrico (RUIZ, BOSCH, GASCÓN, 2015).

O currículo ocidental de Matemática passou por uma reforma na década de 60 que ficou conhecida como o Movimento da Matemática Moderna (MMM). Antes dela, a Álgebra era a porta de entrada para a "Matemática superior". Ao correspondente dos anos iniciais do Ensino Fundamental daquela época cabia apenas a Aritmética; já no equivalente aos anos finais, a Matemática era dividida em Aritmética, Álgebra e Geometria. Essa organização era chamada de "clássica" e era encontrada na maioria dos currículos básicos ocidentais antes do movimento (RUIZ, BOSCH, GASCÓN, 2015). Nessa organização, a iniciação à Álgebra se concentrava em três assuntos: os números algébricos, cálculo de expressões algébricas e equações algébricas. Nesse momento, o currículo ainda guardava um aspecto que Chevallard (1984) enfatiza: a separação e oposição entre a Aritmética e a Álgebra.

A oposição entre esses dois campos é de natureza didática e epistemológica. Na escola ela é muito trabalhada nos anos iniciais e servirá de base para a continuidade do trabalho matemático, incluindo a Álgebra. Historicamente, a Aritmética estava presente no dia a dia, enquanto a Álgebra constituía uma forma de se elevar socialmente. A inovação da Álgebra consistia na introdução dos símbolos algébricos, que funcionava 
como uma espécie de memória da resolução de um problema, permitia conservar o pensamento efetuado. A Álgebra explicitava uma maneira de lidar com números que estava implícita na Aritmética e avançava para problemas complexos, antes limitados a equações do primeiro grau.

O MMM trouxe mudanças ao corpo tradicional da Matemática, a organização clássica foi substituída por tópicos e lições sem agrupamento aparente. A Álgebra sofreu grande redução, sua "parte numérica" resistiu, no entanto, o cálculo e o estudo das equações, que constituíam o coração da Álgebra, sofreram diminuições nos programas após o movimento. Assim, a Aritmética teve seu papel engrandecido, pois a reforma trouxe consigo tendências empiristas, valorizando a Aritmética como a parte "concreta" da Matemática. Na busca entre a teoria e a realidade, a Álgebra, vista como a abstração do real, ficou reduzida e a relação entre os dois campos matemáticos se perdeu.

A partir dos estudos de Chevallard (1984, 1989, 1990), Gascón (1999) traça um modelo dominante do ensino de Álgebra na escola básica que interpreta a Álgebra como Aritmética generalizada. Nela a Álgebra se limita ao contexto numérico.

Interpretar a Álgebra como uma Aritmética generalizada supõe assumir que a Álgebra se constrói em um contexto numérico, como generalização de cálculos com números e tradução de expressões numérico-literais, onde as expressões algébricas surgem da necessidade de representar e manipular números desconhecidos. Portanto, na escrita e manipulação de expressões algébricas, é muito importante distinguir entre dados conhecidos e as incógnitas. Se determinam então como as tarefas mais importantes na Álgebra escolar: a tradução da linguagem natural para a linguagem algébrica, o cálculo algébrico (interpretado como a manipulação formal de regras aritméticas com letras e números) e a resolução de equações. (Tradução nossa, RUIZ, BOSCH, GASCÓN, 2007, p.656)

Nesse modelo, as dificuldades são atribuídas à transição do "pensamento aritmético" para o "pensamento algébrico", reduzindo a Álgebra a uma extensão da Aritmética. Para Gascón (1999), os programas dos anos finais do Ensino Fundamental não são organizados para proporcionar a algebrização progressiva da atividade Matemática. Ela é bruscamente algebrizada quando o currículo não resiste à pressão de certas necessidades que requerem o uso do instrumento algébrico. Assim, a atividade Matemática é extremamente compartimentalizada e acontece um fenômeno chamado 
por Gascón (1999) de "alienação didática", isto é, o aluno passa pela escola sem sentir a necessidade de responder perguntas por ele mesmo elaboradas, sem sentir a necessidade de utilizar a Matemática.

Dessa forma, uma nova proposta precisa enxergar a Álgebra como um instrumento para fazer Matemática. Para isso deve empregar os parâmetros, que permitem estudar a estrutura dos problemas, sem se limitar a descobrir o valor da incógnita.

Ainda que o surgimento da Álgebra se caracterize materialmente pela proliferação de expressões algébricas e pela emergência de uma espécie de "linguagem algébrica", a nova maneira de fazer Matemática baseia sua verdadeira potência nas diversas possibilidades técnicas que surgem do jogo do duplo uso das letras: como "incógnitas" e como "parâmetros". Entre essas possibilidades, destacam-se: resolver simultaneamente uma ampla classe de problemas, justificar, interpretar e controlar o escopo de aplicação das técnicas pré-algébricas (sejam elas "aritméticas", "geométricas" ou "combinatórias") e, além de obter a incógnita quando o problema tem solução, explicar quais são as condições de existência da dita solução e descrever a estrutura do conjunto das soluções." (Tradução nossa, GASCÓN, 1999, p. 80)

Como vimos a Álgebra tem sido, nos últimos anos, condicionada ao contexto numérico, a Aritmética é considerada mais fácil e "concreta", enquanto a Álgebra, abstrata e difícil. Mas, o questionamento que cabe não é a relação fundamental entre a Aritmética e a Álgebra, mas a hierarquia unidimensional estabelecida (RUIZ, BOSCH, GASCÓN, 2015).

Segundo Ruiz, Bosch e Gascón (2015), alguns pesquisadores apontam a algebrização da Arimética e de outras áreas da Matemática, desde os anos iniciais, como um caminho para superar as dificuldades no ensino de Álgebra. Essa proposta chama-se Early Algebra e mostra certa consonância com nossos estudos feitos até aqui, quando propõe uma algebrização da Aritmética.

Assim, a proposta curricular Early Algebra preconiza a "algebrização do currículo" por meio do trabalho com padrões, relações e propriedades matemáticas voltados à estrutura da Matemática e tem a intenção de romper com a ênfase dada a procedimentos de cálculos. Grande parte das pesquisas sobre esta temática tem a Aritmética como 
principal acesso à Álgebra, aproveitando sua presença no currículo e, consequentemente, o conhecimento dos professores a respeito do assunto.

\section{Metodologia}

Para a pesquisa bibliográfica fizemos uma busca no banco de teses e dissertações da Coordenação de Aperfeiçoamento de Pessoal do Nível Superior (Capes) com as seguintes palavras-chave: "early algebra”, "pré-álgebra”, "álgebra nos anos iniciais”, "pensamento algébrico" e "educação algébrica". Com o termo "proto-álgebra" não foram encontrados resultados na busca. Encontramos 152 pesquisas como resultados e identificamos que, dentre essas, 14 se repetiram.

Para organizar os resultados obtidos nas buscas foram lidos os títulos, que muitas vezes já explicitavam o sujeito ou o tema, e sempre que necessário foi consultado o resumo para mais esclarecimentos. Parte dos estudos a respeito do Ensino Fundamental aborda os anos finais (57 pesquisas); um exemplo é a dissertação intitulada 'Resolução de problemas da pré-álgebra e álgebra para fundamental II do ensino básico com auxílio do modelo de barras' (QUEIROZ, 2014).

Outras pesquisas analisam a produção dos alunos ou aplicam sequências de atividades para alunos dos anos iniciais (11 trabalhos), como 'Introdução ao estudo da aritmética e da álgebra no ensino fundamental' (CIVINSKY, 2015). Poucas pesquisas se preocupam com o professor polivalente ( 2 pesquisas), por exemplo 'Álgebra nos Anos Iniciais do Ensino Fundamental: uma análise do conhecimento matemático acerca do Pensamento Algébrico' (FERREIRA, 2017). Os demais (68 trabalhos) possuem outros focos como jogos e História da Matemática; diferentes sujeitos como o professor especialista de Matemática; ou segmentos diferentes da educação como Ensino Superior, Ensino Médio, educação de jovens e adultos, educação inclusiva e educação à distância (FIGURA 2).

Figura 2: Distribuição das pesquisas encontradas 


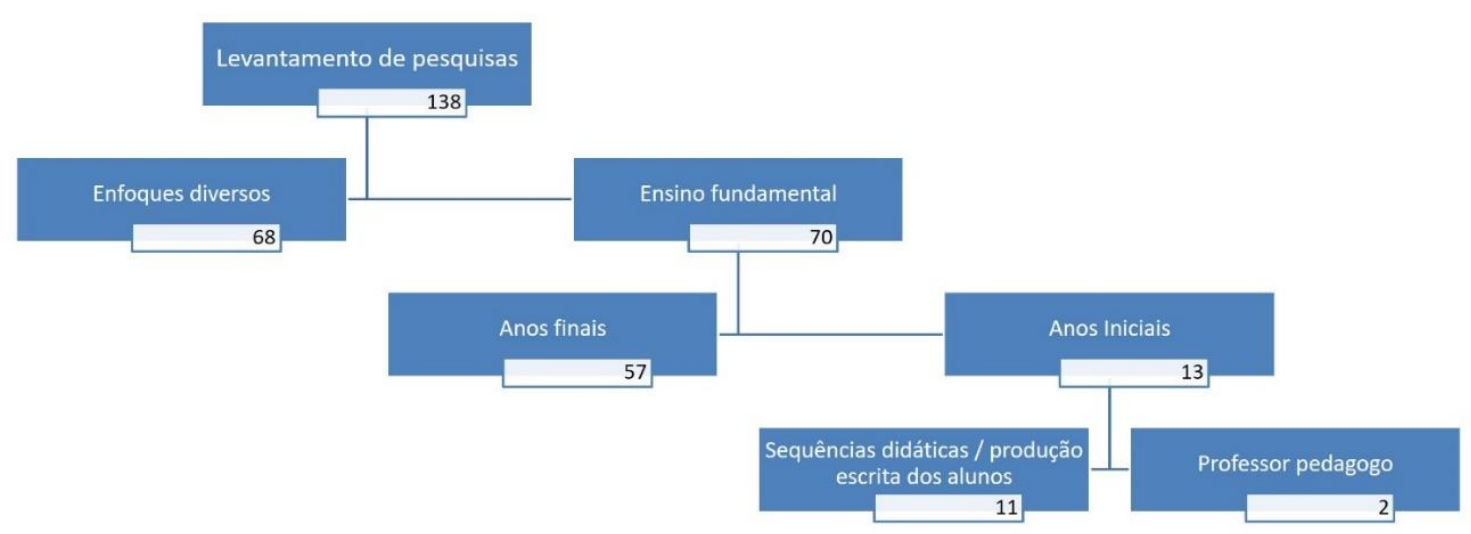

Fonte: elaboração da autora.

Não foram encontradas pesquisas que analisassem conceitos algébricos em materiais didáticos, o que era de se esperar, já que os PCN não dedicavam um eixo à Álgebra nos anos iniciais.

Dessa forma, foram encontrados treze trabalhos que abordam a Álgebra nos anos iniciais, sendo onze sobre sequências didáticas ou a produção escrita dos alunos e dois trabalhos sobre o professor dos anos iniciais.

Tabela1: Pesquisas direcionadas aos anos iniciais do Ensino Fundamental

\begin{tabular}{|c|c|c|c|c|}
\hline ANO & TÍTULO & AUTOR & NÍVEL & CATEGORIA \\
\hline \multirow{3}{*}{2017} & $\begin{array}{l}\text { Álgebra nos anos iniciais do } \\
\text { Ensino Fundamental: uma } \\
\text { análise do conhecimento } \\
\text { matemático acerca do } \\
\text { Pensamento Algébrico } \\
\end{array}$ & $\begin{array}{l}\text { FERREIRA, } \\
\text { Miriam Criez } \\
\text { Nobrega }\end{array}$ & mestrado & $\begin{array}{l}\text { professor } \\
\text { pedagogo }\end{array}$ \\
\hline & $\begin{array}{l}\text { Contribuições dos jogos para } \\
\text { ensinar álgebra nos anos iniciais } \\
\text { do Ensino Fundamental: } \\
\text { perspectivas histórica e atual }\end{array}$ & $\begin{array}{l}\text { ROCHA, Amanda } \\
\text { Moura da }\end{array}$ & mestrado & $\begin{array}{l}\text { sequência } \\
\text { didática }\end{array}$ \\
\hline & $\begin{array}{l}\text { O pensamento algébrico nos } \\
\text { anos iniciais do Ensino } \\
\text { Fundamental: a percepção de } \\
\text { regularidades e o pensamento } \\
\text { relacional }\end{array}$ & $\begin{array}{l}\text { SANTOS, Carla } \\
\text { Cristiane Silva }\end{array}$ & mestrado & $\begin{array}{l}\text { sequência } \\
\text { didática }\end{array}$ \\
\hline 2016 & $\begin{array}{l}\text { A introdução do raciocínio } \\
\text { funcional no } 5^{\circ} \text { ano do Ensino } \\
\text { Fundamental: uma proposta de } \\
\text { intervenção }\end{array}$ & $\begin{array}{l}\text { TEIXEIRA, } \\
\text { Antonio Cesar } \\
\text { Nascimento }\end{array}$ & mestrado & $\begin{array}{l}\text { sequência } \\
\text { didática }\end{array}$ \\
\hline \multirow[t]{2}{*}{2015} & $\begin{array}{l}\text { Introdução ao estudo da } \\
\text { aritmética e da álgebra no } \\
\text { Ensino Fundamental }\end{array}$ & $\begin{array}{l}\text { CIVINSKI, Daiana } \\
\text { Dallagnoli }\end{array}$ & mestrado & $\begin{array}{l}\text { sequência } \\
\text { didática }\end{array}$ \\
\hline & Os problemas aditivos e o & BECK, Vinicius & mestrado & sequência \\
\hline
\end{tabular}




\begin{tabular}{|c|c|c|c|c|}
\hline & $\begin{array}{l}\text { pensamento algébrico no ciclo } \\
\text { da alfabetização }\end{array}$ & Carvalho & & didática \\
\hline \multirow[t]{2}{*}{2014} & $\begin{array}{l}\text { Invariantes operatórios e níveis } \\
\text { de generalidade manifestados } \\
\text { por estudantes dos anos iniciais } \\
\text { do Ensino Fundamental em } \\
\text { tarefas não-rotineiras }\end{array}$ & $\begin{array}{l}\text { BONI, Keila } \\
\text { Tatiana }\end{array}$ & mestrado & $\begin{array}{l}\text { sequência } \\
\text { didática }\end{array}$ \\
\hline & $\begin{array}{l}\text { Manifestação de pensamento } \\
\text { algébrico em registros escritos } \\
\text { de estudantes do Ensino } \\
\text { Fundamental I }\end{array}$ & $\begin{array}{l}\text { FERNANDES, } \\
\text { Renata Karoline }\end{array}$ & mestrado & $\begin{array}{l}\text { produção } \\
\text { escrita dos } \\
\text { alunos }\end{array}$ \\
\hline 2012 & $\begin{array}{l}\text { Caracterizações do pensamento } \\
\text { algébrico em tarefas realizadas } \\
\text { por estudantes do ensino } \\
\text { fundamental I }\end{array}$ & $\begin{array}{l}\text { SILVA, Daniele } \\
\text { Peres da }\end{array}$ & mestrado & $\begin{array}{l}\text { sequência } \\
\text { didática }\end{array}$ \\
\hline 2011 & $\begin{array}{l}\text { Desenvolvimento de conceitos } \\
\text { algébricos por professores dos } \\
\text { anos iniciais do Ensino } \\
\text { Fundamental } \\
\end{array}$ & $\begin{array}{l}\text { FREIRE, Raquel } \\
\text { Santiago }\end{array}$ & doutorado & $\begin{array}{l}\text { professor } \\
\text { pedagogo }\end{array}$ \\
\hline \multirow[b]{2}{*}{2007} & $\begin{array}{l}\text { O que alunos da escola básica } \\
\text { mostram saber por meio de sua } \\
\text { produção escrita em Matemática }\end{array}$ & $\begin{array}{l}\text { SANTOS, João } \\
\text { Ricardo Viola dos }\end{array}$ & mestrado & $\begin{array}{l}\text { produção } \\
\text { escrita dos } \\
\text { alunos } \\
\end{array}$ \\
\hline & $\begin{array}{l}\text { Objetos de aprendizagem para o } \\
\text { desenvolvimento do pensamento } \\
\text { algébrico no Ensino } \\
\text { Fundamental } \\
\end{array}$ & $\begin{array}{l}\text { FREIRE, Raquel } \\
\text { Santiago }\end{array}$ & mestrado & $\begin{array}{l}\text { sequência } \\
\text { didática }\end{array}$ \\
\hline 2001 & $\begin{array}{l}\text { A atribuição de significado em } \\
\text { atividades pré-algébricas por } \\
\text { crianças do } 2^{\circ} \text { ano do } 1^{\circ} \text { ciclo do } \\
\text { Ensino Fundamental }\end{array}$ & $\begin{array}{l}\text { PINTO, Geórgia } \\
\text { Albuquerque de } \\
\text { Toledo }\end{array}$ & mestrado & $\begin{array}{l}\text { sequência } \\
\text { didática }\end{array}$ \\
\hline
\end{tabular}

Fonte: elaboração da autora.

Como não havia nenhum trabalho diretamente relacionado ao nosso tema de pesquisa, escolhemos, então, a dissertação de Ferreira (2017), que tem como sujeito de pesquisa o professor pedagogo, e também analisa a Base Nacional Comum Curricular para os anos iniciais do Ensino Fundamental; e a dissertação de Lima (2018), que tomamos conhecimento por meio do próprio Programa de Estudos Pós-Graduados em Educação Matemática da Pontifícia Universidade Católica de São Paulo, e nos interessa porque analisa documentos curriculares referentes ao ciclo da alfabetização - três primeiros anos do Ensino Fundamental - incluindo a BNCC.

\section{Análises}


A pesquisa de mestrado de Lima (2018) investiga a abordagem do pensamento algébrico no ciclo da alfabetização. Para isso, faz uma análise de conteúdo de dois documentos oficiais, a Base Nacional Comum Curricular (BNCC) e as Orientações Curriculares de Matemática para os Anos Iniciais (OCMAI). O autor identifica o pensamento algébrico no ciclo da alfabetização como a compreensão e identificação de padrões e regularidades que possam ser generalizados em contextos diversos e ainda sem o uso de símbolos algébricos. E aponta que a BNCC explicita a presença de tais conteúdos, enquanto as OCMAI apresentam indícios de um pensamento algébrico desde os anos iniciais, porém de maneira implícita e sem articulação com outras subáreas da Matemática.

A análise de conteúdo realizada pelo autor descreve de maneira objetiva, sistemática e quantitativa o conteúdo dos documentos analisados. O autor cria três categorias de análise. Na primeira categoria, denominada estrutural, o autor observa a dedicação de uma unidade temática para a Álgebra já nos anos iniciais do Ensino Fundamental, o que condiz com a linha de pesquisa Early Algebra. Na segunda categoria, a intramatemática, o autor verifica se a Álgebra permeia outros campos da Matemática, identificando um discurso a respeito da relação entre as unidades temáticas, que aparece de maneira mais sutil no quadro de habilidades proposto para os anos iniciais. Na terceira categoria, denominada conceitual, o pesquisador destaca como principais ideias a serem trabalhadas nos três primeiros anos do Ensino Fundamental: regularidades; generalização de padrões; propriedades da igualdade; equivalência; e relação entre variáveis, fazendo uso de símbolos, mas sem a exigência do formalismo algébrico. $\mathrm{O}$ autor conclui que a concepção de ensino de Álgebra apresentada para os anos iniciais na BNCC é bastante próxima da corrente Early Algebra, e salienta que, apesar de a BNCC declarar a unidade temática Álgebra, há conceitos algébricos em outras unidades temáticas também, articulando a Álgebra com outros campos da Matemática.

A segunda pesquisa é a dissertação de Ferreira (2017), organizada no formato multipaper, composta por três artigos independentes, cada um com um objetivo específico, que se articulam para atingir o objetivo geral da pesquisa: investigar o conhecimento matemático para a abordagem do pensamento algébrico nos anos iniciais do Ensino Fundamental.

O primeiro artigo consiste na análise de documentos curriculares e documentos que orientam a formação de professores. Foram levantadas as categorias mais comumente 
trabalhadas nos anos iniciais - Aritmética Generalizada e Pensamento Funcional - e suas subcategorias, a partir da pesquisa de Blanton e Kaput (2005). A partir da análise documental, a autora conclui que diretrizes curriculares mais antigas tem maior enfoque nas propriedades dos números e das operações dentro da categoria Aritmética Generalizada, deixando de lado outras subcategorias da mesma e a categoria Pensamento Funcional. Em 2012 surge, com os Direitos de Aprendizagem do PNAIC (Pacto Nacional pela Alfabetização na Idade Certa), um eixo específico para o pensamento algébrico, porém com o trabalho mais voltado para padrões. A autora conclui que, no geral, são poucas subcategorias do pensamento algébrico nos currículos oficiais, sendo a primeira versão da BNCC, o documento que trata com maior intencionalidade e abrange o maior número das subcategorias elencadas pela autora. Documentos anteriores, incluindo os Parâmetros Curriculares Nacionais, tinham o trabalho mais voltado para a categoria Aritmética Generalizada.

O segundo e o terceiro artigos foram elaborados a partir de uma formação continuada, respectivamente, com os objetivos de identificar qual a compreensão de professores dos anos iniciais acerca do significado do pensamento algébrico e em que medida eles reconhecem os elementos que o constituem; e identificar os conhecimentos matemáticos revelados por um grupo de professores dos anos iniciais quando discutem tarefas com potencial algébrico. A autora conclui que, no que concerne ao pensamento algébrico, os professores têm um conhecimento mais metodológico e menos voltado ao conteúdo. Já no terceiro artigo conclui que o conhecimento dos professores acerca do pensamento algébrico está muito relacionado ao saber fazer, mas não alcança os porquês matemáticos associados ao que se faz.

\section{Considerações Finais}

Percebemos que há uma mudança no cenário acerca do pensamento algébrico a ser trabalhado nos anos iniciais. Já existem algumas pesquisas preocupadas com esse tema no campo da Educação Matemática. Conforme as pesquisas citadas (LIMA, 2018; FERREIRA, 2017), as mudanças já são percebidas nos documentos oficiais, principalmente na BNCC. As conclusões de Lima (2018) apontam para a necessidade de analisar todas as habilidades dos anos iniciais e não só as habilidades pertencentes a unidade temática Álgebra. A pesquisa de Ferreira (2017) analisa a primeira versão da BNCC de uma maneira próxima da que pretendemos analisar em pesquisas futuras. 
É intenção dos autores dar continuidade à análise de Ferreira (2017), utilizando a versão homologada da BNCC, e verificar se todas as habilidades propostas no documento tem correspondência com as categorias elencadas a partir de Blanton e Kaput (2005). Para, então, analisar livros didáticos dos anos iniciais do Ensino Fundamental elaborados antes e depois da homologação da BNCC com o objetivo de verificar como estas mudanças estão sendo incorporadas aos materiais didáticos.

\section{Agradecimentos}

À Coordenação de Aperfeiçoamento de Pessoal de Nível Superior (CAPES) pela bolsa de estudos concedida.

\section{Referências}

BECK, V. C. Os problemas aditivos e o pensamento algébrico no ciclo da alfabetizaçãa. 2015. Dissertação (Mestrado em Educação) - Programa de PósGraduação em Educação, Universidade Federal do Rio Grande, Rio Grande, 2015.

BLANTON, M. L.; KAPUT, J. J. Characterizing a classroom practice that promotes algebraic reasoning. Journal for Research in Mathematics Education, v. 36 n.5, p. 412-446, 2005.

BONI, K.Ta. Invariantes operatórios e níveis de generalidade manifestados por estudantes dos anos iniciais do Ensino Fundamental em tarefas não-rotineiras. 2015. Dissertação (Mestrado em Ensino de Ciências e Educação Matemática) Programa de Pós-Graduação em Ensino de Ciências e Educação Matemática, Universidade Estadual de Londrina, Londrina, 2014.

BRASIL. Ministério da Educação, Secretaria de Educação Fundamental. Parâmetros curriculares nacionais. Matemática: Ensino de primeira à quarta série. Brasília: 1997.

BRASIL. Ministério da Educação. A Base Nacional Comum Curricular. Brasília: 2017.

BRASIL. Ministério da Educação. Guia de implementação da Base Nacional Comum Curricular: orientações para o processo de implementação da BNCC. Brasília: 2018.

CHEVALLARD, Y. Le passage de l'arithmetique a l'algebrique dans l'enseignement des mathematiques au college. Preimiere partie: L' évolution de la transposition didactique. Petit x, n. 5, p. 51-94, 1984.

Le passage de l'arithmetique a l'algebrique dasn l'ensegnement des mathematiques au college. Deuxeieme partie, perspectives curriculares : la notion de modelisation. Petit x, n. 19, p. 43-72, 1989.

. Lepassage de l'arithmetique a l'algebrique dans l'enseignement des mathematiques au college. Troisième partie. Voies d'attaque et problemas didactiques. Petit x, n. 23, p. 5-38, 1990.

CIVINSKI, D. D. Introdução ao estudo de aritmética e da álgebra no ensino fundamental. Dissertação (Mestrado profissional em ensino de ciências naturais e 
matemática) - Programa de Pós-Graduação em Ensino de Ciências Naturais e Matemática, Universidade Regional de Blumenau, Blumenau, 2015.

FERNANDES, R. K. Manifestação de pensamento algébrico em registros escritos de estudantes do Ensino Fundamental I. 2014. Dissertação (Mestrado em Ensino de Ciências e Educação Matemática) - Programa de Pós-Graduação em Ensino de Ciências e Educação Matemática, Universidade Estadual de Londrina, Londrina, 2014.

FERREIRA, M. C. N. Álgebra nos anos iniciais do ensino fundamental: uma análise do conhecimento matemático acerca do Pensamento Algébrico. 2017. Dissertação (Mestrado em ensino e história das ciências e da matemática) - Programa de PósGraduação em Ensino e História das Ciências e da Matemática, Universidade Federal do ABC, Santo André, 2017.

FREIRE, R. S. Objetos de aprendizagem para o desenvolvimento do pensamento algébrico no Ensino Fundamental. 2007. Dissertação (Mestrado em Educação Brasileira) - Programa de Pós-Graduação em Educação Brasileira, Universidade Federal do Ceará, Fortaleza, 2007.

Desenvolvimento de conceitos algébricos por professores dos anos iniciais do Ensino Fundamental. 2011. Tese (Doutorado em Educação Brasileira) - Programa de Pós-Graduação em Educação Brasileira, Universidade Federal do Ceará, Fortaleza, 2011.

GASCÓN, J. La naturaleza prealgebraica de la matemática escolar. Educación Matemática, v. 11, n. 1, p. 77-88, 1999.

KATZ, V. J. The development of álgebra and álgebra education. In: LACAMPAGNE, C.; BLAIR, W.; KAPUT, J. (Eds.): The álgebra initiative colloquium, Vol 1. Washington, DC: US Department os Education, 1995.

LIMA, J. R. de C. Pensamento algébrico no currículo do ciclo de alfabetização: estudo comparativo de duas propostas. 2018. Dissertação (Mestrado em educação matemática) - Programa de Estudos Pós-Graduados em Educação Matemática, Pontifícia Universidade Católica de São Paulo, São Paulo, 2018.

QUEIROZ, J. M. dos S. Resolução de problemas da pré-álgebra e álgebra para fundamental II do ensino básico com auxílio do modelo de barras. 2014 Dissertação (Mestrado profissional em ensino de ciências exatas) - Programa de Pós-Graduação em Ensino de Ciências Exatas da Universidade Federal de São Carlos, 2014.

ROCHA, A. M. da. Contribuições dos jogos para ensinar álgebra nos anos iniciais do Ensino Fundamental: perspectivas histórica e atual. 2017. Dissertação (Mestrado em Educação em Ciências e Matemáticas - Área de Concentração em Educação Matemática) - Programa de Pós- Graduação em Educação em Ciências e Matemáticas do Instituto de Educação Matemática e Científica, Universidade Federal do Pará, Pará, 2017.

RUIZ, N., BOSCH, M., GASCÓN, J. La algebrización de los programas de cálculo aritmético y la introducción del álgebra en secundaria. In: BRONNER, A.; LARGUIER, M.; ARTAUD, M.; BOSCH, M.; CHEVALLARD, Y.; CIRADE, G.; LADAGE, C. (Éds). II congrès international sur la TAD, p. 655-676, 2007.

. La algebrización de los programas de cálculo aritmético y la introducción del álgebra en secundaria. In: MORENO, M.M.; ESTRADA, A.; CARRILLO, J.; SIERRA, T.A. (Eds.). Investigación en Educación Matemática XIV. Lleida: SEIEM, 2010. p. 
545-556).

El problema Didáctico del Álgebra Elemental: Un Análisis Macro-Ecológico

desde la Teoría Antropológica de lo. REDIMAT, v. 4, n. 2, p. 106-131, 2015.

SANTOS, J. R. V. dos. O que alunos da escola básica mostram saber por meio de sua produção escrita em Matemática. 2007. Dissertação (Mestrado em Ensino de Ciências e Educação Matemática) - Programa de Pós-Graduação em Ensino de Ciências e Educação Matemática, Universidade Estadual de Londrina, Londrina, 2007.

SANTOS, C. C. S. O pensamento algébrico nos anos iniciais do Ensino

Fundamental: a percepção de regularidades e o pensamento relacional. 2017.

Dissertação (Mestrado em Educação) - Programa de Pós-Graduação Stricto Sensu em Educação, Universidade São Francisco, Itatiba, 2017.

SILVA, D. P. da. Caracterizações do pensamento algébrico em tarefas realizadas por estudantes do ensino fundamental I. 2013. Dissertação (Mestrado em Ensino de Ciências e Educação Matemática) - Programa de Pós-Graduação em Ensino de Ciências e Educação Matemática, Universidade Estadual de Londrina, Londrina, 2013.

TEIXEIRA, A. C. N. A introdução do raciocínio funcional no $5^{\circ}$ ano do Ensino Fundamental: uma proposta de intervenção. 2016. Dissertação (Mestrado em Educação Matemática) - Programa de Pós-Graduação em Educação Matemática, Universidade Estadual de Santa Cruz, Ilhéus, 2016. 